\title{
Das historische Porträt: Professor Dr. Werner Schmidt (1907-1989) und die flussüberschreitende Dermatologie im Rhein-Neckar-Dreieck ${ }^{1}$
}

\author{
Historical Portrait: Professor Dr. Werner Schmidt (1907-1989) and Dermatology in the Rhine-Neckar-Triangle
}

Autor

Institut

\section{Löser}

Hautklinik Ludwigshafen

\section{Bibliografie}

DOI http://dx.doi.org/

10.1055/s-0029-1215409

Online-Publikation: 1. 12. 2009

Akt Dermatol 2010; 36:

14-17 @ Georg Thieme

Verlag KG Stuttgart · New York ISSN 0340-2541

\section{Korrespondenzadresse}

Dr. Christoph Löser

Leitender Oberarzt,

Hautklinik Ludwigshafen

(Direktor Prof. Dr. Edgar Dippel)

Klinikum Ludwigshafen gGmbH

Bremserstr. 79

67063 Ludwigshafen

christoph.loeser@klilu.de

\section{Zusammenfassung \\ $\nabla$}

Unter Werner Schmidt wurde die Hautabteilung am Städtischen Krankenhaus in Ludwigshafen zur Hautklinik und die Hautklinik in Mannheim zur universitären Einrichtung. Die Chefarztposition in Ludwigshafen wurde wiederum in seiner

\section{Einleitung}

Dieser Artikel erinnert an Professor Dr. Werner Schmidt, eine historische Persönlichkeit der Dermatologie. Außerdem geht es um seinen prägenden Einfluss auf zwei benachbarte dermatologische Klinikstandorte - Ludwigshafen und Mannheim $($ Abb. 1).

\section{Ludwigshafen und Mannheim \\ $\nabla$}

Fragt ein Unkundiger auf einem internationalen Kongress nach der Lage der Stadt Ludwigshafen, so führt der naheliegende Hinweis „close to Mannheim“ kaum weiter. Denn den Ludwigshafenern zum Trost ist Mannheim dann ebenso unbekannt. Häufig hilft erst die Nennung von Frankfurt bei der geografischen Einordnung. Dem landeskundigen Leser ist Mannheim selbstverständlich ein Begriff, zumindest architektonisch als „Quadratestadt“ oder als ICE-Haltepunkt. Ludwigshafen, die Industriestadt mit dem Chemiegiganten BASF, liegt auf der anderen, der rheinlandpfälzischen Rheinseite und ist entstanden aus der so genannten Rheinschanze, ursprünglich eine Festungsanlage zum Schutze Mannheims, und den umliegenden Ortschaften, von denen die ehemals kurfürstliche Stadt Oggersheim spä-

\footnotetext{
${ }^{1}$ Herrn Dr. Klaus Holzegel anlässlich der Verleihung der Silbernen Ehrennadel der Ärztekammer Sachsen-Anhalt $12 / 2009$.
}

Nach-Nachfolge aus der Mannheimer Klinik von Professor Volker Voigtländer (1990-2008) besetzt. Dessen Nachfolger und heutiger Amtsinhaber, Professor Edgar Dippel, hatte wiederum zwischenzeitlich in Mannheim gewirkt. Und so zeigt sich eine Nähe der beiden Städte nicht nur geografisch, sondern auch in der Dermatologie.

ter als Wohnort des damaligen Bundeskanzlers Dr. Helmut Kohl internationale Bekanntheit erlangte. Beide Städte besitzen kulturelle Attraktionen wie Orchester und Theater, Museen und Parkanlagen sowie überregional bekannte Kliniken. Sie teilen sich den Fluss und nähern sich heute als Zentrum der Metropolregion Rhein-Neckar immer weiter an. Weniger geläufig sind die Verbindungen in der Dermatologie, die mit Professor Werner Schmidt begannen.

\section{Der Werdegang von Werner Schmidt}

Werner Schmidt ( $\bullet$ Abb.2) wurde am 9. April 1907 in Saarbrücken geboren. Er hatte eine ältere Schwester. Sein Vater Hans war kaufmännischer Direktor des Arbed-Konzerns, einer luxemburgischen Gesellschaft, zu der auch die Burbacher Hütte gehörte, deren Direktor einmal der spätere Schwiegervater von Werner Schmidt werden sollte.

Im Alter von 15 Jahren zog seine Familie nach Stuttgart, wo er am Realgymnasium 1926 das Abitur machte. Er studierte anschließend in Tübingen, Würzburg, Hamburg, Berlin und wieder Tübingen. Dort beendete er sein Medizinstudium 1931 mit dem Staatsexamen. Auf die Promotion folgte 1932 die Medizinalpraktikantenzeit in Plauen i.V., Baden-Baden und Stuttgart. Seine Weiterbildung begann er 1933 in Essen bei dem Dermatologen Alois Memmesheimer (18941973) und wechselte 1934 zurück nach Tübingen 


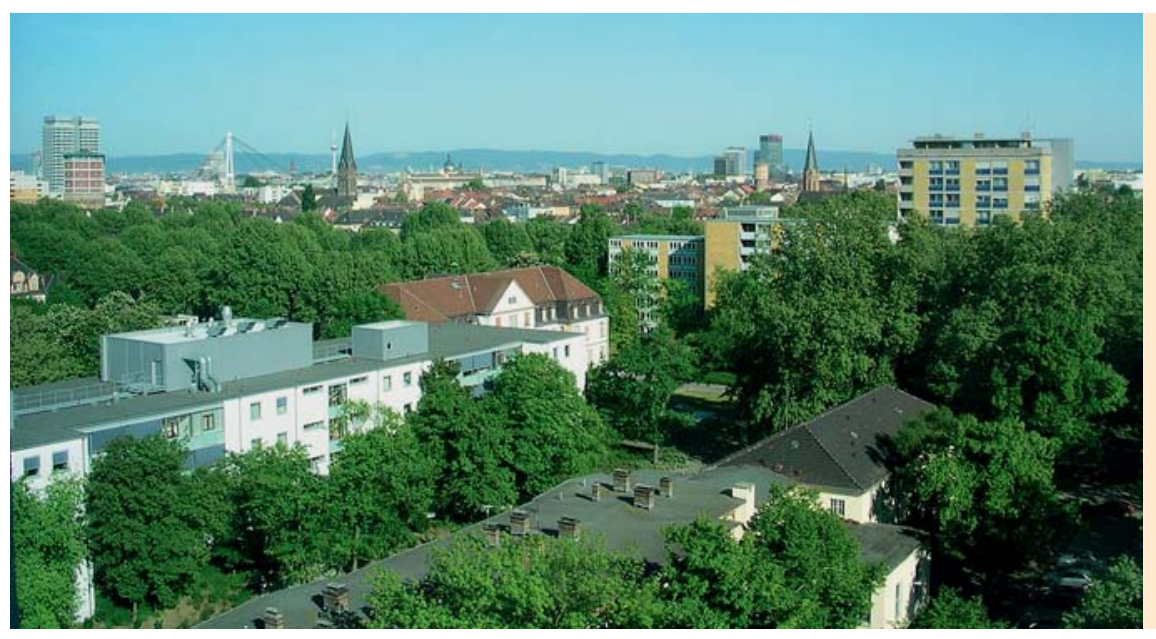

Abb. 1 Blick auf die heutige Hautklinik Ludwigshafen (langgestrecktes Gebäude unten links) und über Ludwigshafen und Mannheim in Richtung Osten. Die Hautklinik Mannheim liegt links zwischen Rheinbrücke und Fernsehturm, im Hintergrund der Odenwald.

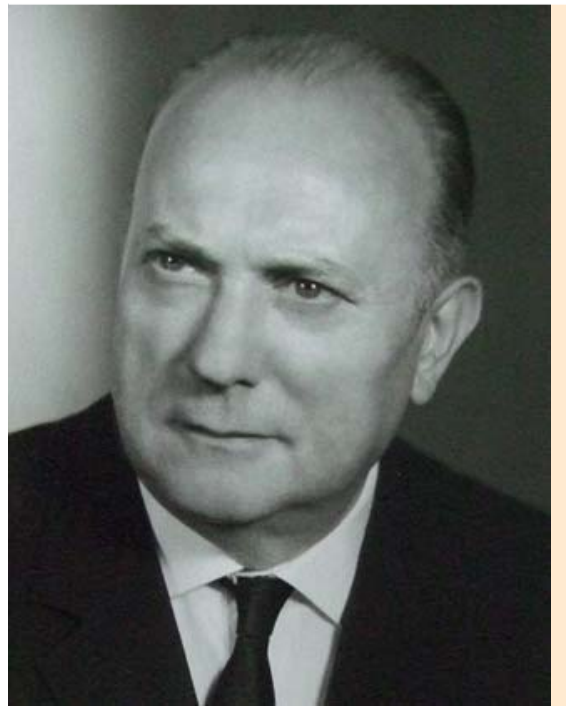

Abb. 2 Porträt Prof. Dr. Werner Schmidt (1907-1989), undatiert.

zu Paul Linser (1871 - 1963). 1935 ging er als Oberarzt zu Heinrich Löhe (1877-1961) nach Berlin. Dort habilitierte er sich 1939 über „Das Vorkommen von regionären Verschiedenheiten und zeitlichen Schwankungen im Reaktionsausfall intracutaner Allergen-Injektionen“. Im gleichen Jahr wurde er zum Militär eingezogen, nahm am Polenfeldzug teil und kehrte 1941 als „unabkömmlich“ nach Berlin zurück. Er leitete dann die Hautklinik am Rudolf-Virchow-Krankenhaus in Vertretung von Heinrich Löhe, der als Generalarzt bei der Wehrmacht die Dermatologie vertrat. Eine Stelle als Chefarzt der Hautklinik in Posen durfte er 1942 nicht antreten, weil der Magistrat der Stadt Berlin die Entlassung aus seiner Dienstverpflichtung verweigerte. Nach Kriegsende konnte er sich „Anwerbungsversuchen“ nach Russland entziehen und dank eines alten Tübinger Passes zu seiner Familie nach Stuttgart durchschlagen.

1946 übernahm er zunächst die ärztliche Leitung der Fachabteilung für geschlechtskranke Frauen in Wimbern an der Ruhr. Diese im Krieg von der Organisation Todt als Barackenkrankenhaus errichtete, architektonisch interessante Klinik war ein Ausweichkrankenhaus der Stadt Dortmund, unterstand aber dem Klinikum in Münster. Mit der Verfügbarkeit des Penizillins änderte sich der stationäre Behandlungsbedarf schlagartig und Werner Schmidt eröffnete daraufhin 1946 seine eigene Hautarztpraxis in Stuttgart. Zeitgleich war er bis 1948 kommissarischer Leiter der Hautabteilung in Ludwigsburg, solange deren Leiter, ein Namensvetter, in einem Entnazifizierungsverfahren gebunden war. $[1,5]$

Orte wie Ludwigshafen sind die ersten Seestädte auf dem Land, fluktuierend, aufgelockert, am Meer einer unstatischen Zukunft.

Ernst Bloch

\section{Dermatologie in Ludwigshafen}

Die Dermatologische Abteilung am Städtischen Krankenhaus in Ludwigshafen war 1910 gegründet worden. Siegfried Fuss leitete die Abteilung 40 Jahre lang mit einem geringen Entgelt der Stadt und betrieb eine große Kassenarztpraxis. Auf die 1949 ausgeschriebene Stelle gab es 15 Bewerbungen, darunter die von Dozent Dr. Werner Schmidt und anderen bekannten Namen wie Prof. Dr. Julius Dörffel, Prof. Dr. Josef Vonkennel, Prof. Dr. Hans Dölken und Prof. Dr. Ferdinand John. In einer Sitzung des Personalausschusses der Stadt Ludwigshafen wurde 1950 die Neubesetzung verhandelt. Im Protokoll dieser Sitzung findet sich die Einschätzung, „dass die Mannheimer und Heidelberger Krankenhäuser eine schwere Konkurrenz bedeuten und in Ludwigshafen immer mit wenig Privatpatienten zu rechnen sein wird. Der Chefarzt der Dermatologischen Abteilung werde deshalb in der Hauptsache Kassenpraxis betreiben müssen “. Deshalb wurde in der gleichen Sitzung die Auffassung vertreten, dass sich die Stadt bei der Wahl eines Mannheimer Bewerbers vermutlich die Zuweisung einer Wohnung erspare. Dennoch setzte sich Werner Schmidt schließlich durch. Im Protokoll ist dazu vermerkt: „Der Bewerber stamme aus Zweibrücken und sei 10 Jahre am größten deutschen Krankenhaus in Berlin als Dermatologe tätig gewesen. Seine Zeugnisse seien ausgezeichnet, die Auskünfte über ihn, auch von Außenstehenden, hervorragend. Seine Ausbildung übersteige weit das normale Maß. Er halte ihn deshalb für den geeignetsten und würdigsten Bewerber...“

1950 trat Werner Schmidt seine Stelle als Leiter der Dermatologischen Abteilung am Städtischen Krankenhaus Ludwigshafen an. Die Abteilung verfügte über 96 Betten. Auch gab es die damals üblicherweise geschlossene Station für Geschlechtskranke. Der Bürgermeister hatte die Räume eigens neu streichen lassen und interessierte sich bei seinem Begrüßungsbesuch offenbar mehr für die Lackierarbeiten als für die medizinische Ausstattung. Die 
schwierigen Zustände der Nachkriegszeit mit den verbliebenen Bombenschäden wurden auch durch die Matratzen augenfällig, die nicht in die vorhandenen Bettgestelle passten. Nach einer finanziell stark eingeschränkten Anfangszeit wurde Werner Schmidt verbeamtet und konnte ab 1952 eine Privatambulanz betreiben. Im gleichen Jahr habilitierte er sich nach Mainz um und wurde 1956 zum außerplanmäßigen Professor ernannt. Zweimal wöchentlich fuhr er dann nach Mainz, um Vorlesung zu halten - seine Frau begleitete ihn auf diesen Fahrten und sorgte als seine Vorlesungsassistentin für eine saubere Tafel und frische Kreide. Unter seinen Hörern waren damals viele ausländische Studenten, meist mit Ausbildung in England, die sich bei der Berechnung der komplizierten dermatologischen Rezepturen offenbar vielfach leichter taten als ihre deutschen Kommilitonen. Im Jahr 1957 wurde die Dermatologische Abteilung in Ludwigshafen unter Werner Schmidt dann zur Hautklinik. Im Mittelpunkt stand die konservative Behandlung von Hauterkrankungen. Operationen, die heute einen wesentlichen Schwerpunkt darstellen, wurden nicht durchgeführt. $[1,2,12]$

Junge Städte bieten für Medizinhistoriker nur selten eine ergiebige Fundgrube. Mannheim wurde 1607 gegründet, ist also noch eine junge Stadt.

Werner Schmidt

\section{Werner Schmidt in Mannheim \\ $\nabla$}

1958 wurde Werner Schmidt in der Nachfolge von Friedrich Schmidt-La Baume Direktor der Hautklinik in Mannheim. Unter seiner Leitung wurden die Krankensäle zu kleineren Krankenzimmern umgebaut. Ein wichtiges Verdienst war die Schaffung eines Operationsraumes für dermatologische Eingriffe. Dafür holte er Johannes Petres (1934 - 2007), den später renommierten Dermatochirurgen und Chef der Hautklinik in Kassel, als Oberarzt nach Mannheim.

Nachdem Mannheim 1964 medizinische Fakultät geworden war, folgte 1966 für Werner Schmidt der Lehrauftrag für Dermatologie und Venerologie und 1968 die ordentliche Professur. Der Lehrbetrieb musste von Null aufgebaut werden. Dazu gehörte auch die Schaffung einer dermatologischen Bibliothek. Außerdem widmete er sich der Venerologie ab 1971 als Vorsitzender der „Gesellschaft für Sexualerziehung und zur Bekämpfung der Geschlechtskrankheiten in Baden-Württemberg“. Am 5. November 1975 hielt er seine akademische Abschiedsvorlesung mit anschließender Emeritierungsfeier $(\bullet$ Abb. 3,4$)$. Von seinen Studenten wurde er mit einem traditionellen Fackelzug verabschiedet. [1,6-9]

\section{Werner Schmidt, der Dermatologe und Mensch $\nabla$}

Als Dermatologe setzte sich Werner Schmidt klinisch und wissenschaftlich vor allem mit der Allergologie und Berufsdermatosen auseinander. Letzteres sicher begünstigt durch den Industriestandort Ludwigshafen. In fast einhundert Publikationen beschäftigte er sich vor allem mit Themen wie der Hyposensibilisierung, der Prävention durch Allergiekarenz und dem Hautschutz, außerdem widmete er sich den Geschlechtskrankheiten und den männlichen Fertilitätsstörungen. Der therapeutische Einsatz von oralen Antihistaminika zur Behandlung allergischer Erkrankungen wurde von Werner Schmidt in Deutschland bekannt gemacht.

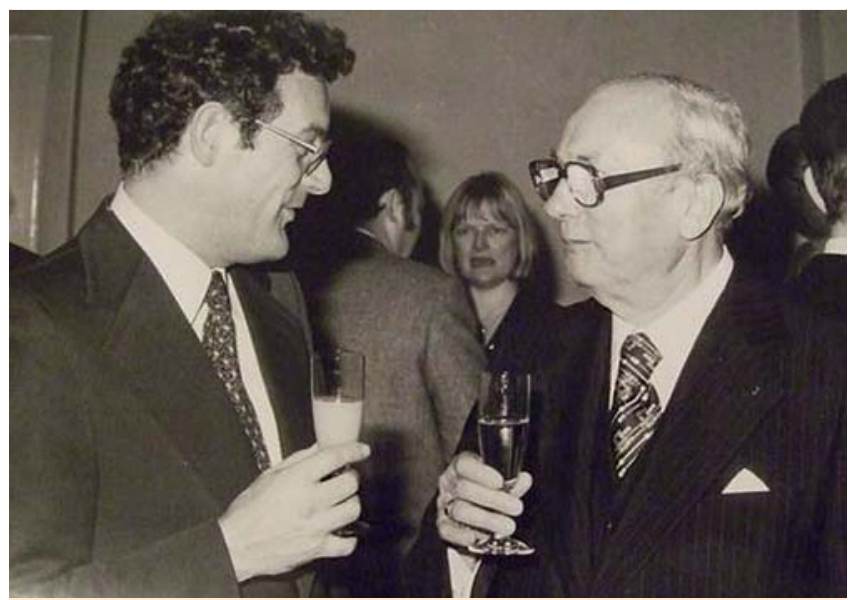

Abb. 3 Werner Schmidt bei einem Empfang mit seinem Nachfolger Prof. Dr. Ernst G. Jung, undatiert.

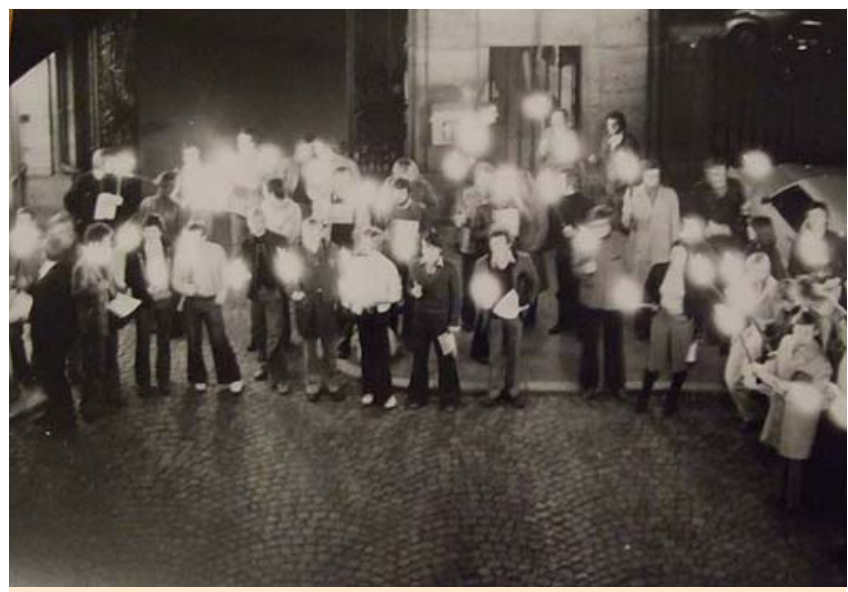

Abb. 4 Fackelzug der Studenten nach der Abschiedsvorlesung 1975, im Hintergrund das historische Tor des Mannheimer Klinikums.

1940 hatte er auf Veranlassung der Elternpaare, die sich gut kannten, in Berlin die 13 Jahre jüngere Liesel (Elisabeth) Nohl kennengelernt, die in Wien und Luxenburg aufgewachsen war und im Lettehaus für Fotografie eine Ausbildung absolvierte. Sie heirateten 1942 und bekamen 1943 das erste von vier Kindern, einen Sohn, der Lehrer wurde. 1944 und 1946 folgten zwei Mädchen, die in Heidelberg eine MTA-Ausbildung absolvierten, und 1955 ein weiteres Mädchen, welche Juristin wurde.

In Ludwigshafen wohnte die Familie im Stadtteil Friesenheim nahe der Klinik zunächst in den Aspen, dann in der Erzbergerstraße am Ebertpark. Aufgrund der seinerzeit üblichen Residenzpflicht erfolgte mit dem Wechsel nach Mannheim der Umzug in den Stadtteil Feudenheim.

Werner Schmidt war ein ausgewiesener Weinkenner und -liebhaber, was sich besonders gut mit der Zeit in Ludwigshafen und der Nähe zur Pfalz vertrug. Über seinen Schwiegervater bestanden persönliche Kontakte zu Winzern. Und abends zog er sich offenbar gerne mit einer guten Flasche Weißwein des Weingutes Bassermann-Jordan in sein Arbeitszimmer zurück, um sich wissenschaftlichen Themen zu widmen. Erst ab 22 Uhr begann dann der gemütliche Teil des Familienlebens. Außerdem war er historisch interessiert. Davon zeugen seine Beschäftigung mit der Deutschen Territorialgeschichte, der Geschichte der Dermatologie und insbesondere der Medizingeschichte Mannheims. 
Entgegen dem Wunsch und der Empfehlung der Medizinhistoriker publizierte er seine Texte zur Mannheimer Medizingeschichte allerdings nicht in renommierten Fachzeitschriften, sondern für seine Studenten in deren Studentenzeitung „Mannheimer MED-Journaille“.

Am 14. August 1989 verstarb Werner Schmidt im Alter von 82 Jahren in Baden-Baden an einem Lymphom. [3-11]

\section{Danksagung}

Herrn Professor Dr. Ernst G. Jung danke ich für Anregung, Ansporn und Unterstützung. Er hat außerdem den Kontakt zu Frau Elisabeth Schmidt vermittelt, die heute in Schlewig-Holstein lebt und der ich für den netten telefonischen Austausch mit vielen persönlichen Erinnerungen und Anekdoten herzlich danke. Herr Dr. Stefan Moerz vom Stadtarchiv Ludwigshafen, Frau Dr. C. Wagner und Herr Dr. Wolfgang Koenen von der Klinik für Dermatologie, Venerologie und Allergologie in Mannheim sowie Herr Professor Dr. Volker Voigtländer, Heidelberg, standen bei der Materialsuche hilfreich zur Seite.

\section{Abstract}

\section{Historical Portrait: Professor Dr. Werner Schmidt (1907 - 1989) and Dermatology in the Rhine-Neckar- Triangle}

Under the auspices of Werner Schmidt (1907 - 1989) the department of Dermatology at the municipal hospital of Ludwigshafen became skin hospital and later the skin hospital of Mannheim became part of the University. One of his successors in Ludwigshafen was Volker Voigtländer (1990 - 2008), who came from Mannheim and was followed by Edgar Dippel, who also spent time in Mannheim and is head today. This illustrates the closeness of both cities not just geographically but in Dermatology as well.

\section{Literatur}

1 Gartman H. Werner Schmidt zum 65. Geburtstag. Hautarzt 1972; 23: $190-191$

2 Hippel von W. Zum Wohle der Kranken. Vom Hospital zum Klinikum der Stadt Ludwigshafen am Rhein. Ludwigshafen: Verlag Regionalkultur, 1992

3 Jung EG. Werner Schmidt zum 70. Geburtstag. Mannheimer Morgen, 12. April 1977

4 Jung EG. Professor Werner Schmidt zum 75. Geburtstag. Heidelberger Tageblatt, 8. April 1982

5 Jung EG. In Memoriam Werner Schmidt 1907-1989. Hautarzt 1989; 40: 789

6 Lischka G. Zur Emeritierung Werner Schmidts. Z Hautkr 1975; 51: 1-2

7 Lischka G. Emeritierungsfeier für Professor Dr. Werner Schmidt in Mannheim. Hautarzt 1976; 27: 506

8 Memmesheimer A. Zum 65. Geburtstag von Werner Schmidt. Z HautGeschl Kr 1972; 7: 276

9 Mit Fackelzug verabschiedet. Professor Werner Schmidt hielt seine letzte Vorlesung. Mannheimer Morgen, 6. November 1975

10 Seine Vorlesungen waren gefragt. Dermatologe Professor Dr. Werner Schmidt feierte 75. Geburtstag. Mannheimer Morgen, 13. April 1982

11 Der Dermatologe Schmidt wird heute 80 Jahre alt. Mannheimer Morgen, 9. April 1987

12 Voigtländer $V$, Bolet $W$, Tully G. Vom Krätzezimmer zur modernen Hautabteilung. Hautarzt 2000; 51: 957-960 